\title{
New countermeasures considered as drug counterfeiting grows
}

With sales of counterfeit medicines apparently on the rise, experts in security and encryption met to discuss possible new solutions in early June at the Fourth Global Forum on Pharmaceutical Anti-counterfeiting held in Washington, DC.

According to the World Health Organization, estimates for the percentage of counterfeit drugs range from around $1 \%$ in developed countries-where they have reached pharmacy shelves-to more than $10 \%$ in developing countries. These fake medicines sport labels that deliberately conceal their true source or identity, and they often contain inactive or harmful ingredients.

Seizures of counterfeit drugs have risen rapidly in Europe, with 4,081,056 packages discovered in 2007, up from 560,598 in 2005. "Most public health experts, customs officers and health regulators say this is a creeping problem," says Jim Thomson, chairman of the London-based European Alliance for Access to Safe Medicines.

At the recent meeting in Washington companies plied their array of barcodes, magnetic tags, and three-dimensional and reflective images, some identifiable only with proprietary scanners. Among the attendees was Randall Burgess, a manager with Tesa Scribos, which markets secure authenticity systems to makers of luxury goods such as handbags. At his booth, Burgess showed off the company's crown jewel: a tiny label sporting four layers of security, including miniscule digits detectable with a magnifying glass and a hologram interpretable only with a laser reader he pulled from his pocket.

Labeling systems such as this could provide unique codes for each drug packet and help establish online tracking systems to trace where each package has been manufactured, packaged and distributed. Such measures could help shore up weak points in the supply chain where counterfeits enter the marketsuch as fake pills sold to drug distributors, a common mechanism.

But with so many technological optionsand a hodgepodge of laws requiring the use of such measures - pharmaceutical companies have been clamoring for standardization.
Many have turned to GS1, the international organization behind the universal bar code used, for example, to ring up products at the supermarket checkout aisle.

"We want to create a guide that you can take to your company or your drug regulators that says: this is how we can do it," Tim Marsh, who co-chairs the arm of GS1 that is working on the problem, said at the recent meeting. (Marsh also serves as senior manager of Global Packaging Technology at Pfizer.)

Some experts say the only way to truly secure the drug supply is with counterfeitproof markings on each pill. That challenge appeals to Facundo Fernandez, an investigator from the Georgia Institute of Technology in Atlanta, who attended the recent meeting in Washington.

Fernandez is designing a portable device that detects a chemical label etched into a pill. Such a label, he envisions, could consist of the active ingredient in the pill labeled with a distinct isotope of carbon easily identified by mass spectroscopy.

Charlotte Schubert, Washington, DC

\section{Victims of West Nile virus face long-term health problems}

Severe infection with West Nile virus is a spreading problem worldwide that disproportionately affects those over age 50 . And evidence is mounting that when the virus causes sickness, the long-term heath effects can be serious.

A close follow-up with 172 patients with confirmed West Nile virus infection published in April suggests that people who have symptomatic infections often suffer lifetime consequences that include memory problems, loss of balance and tremors (Vector Borne Zoonotic Dis. 8, 167-174; 2008).

Among those with severe infections, 60\% still suffered from persistent symptoms of West Nile infection after the end of the first year of infection. Moreover, the researchers discovered that most, if not all, recovery takes place in the first two years after infection. "If a patient has not recovered by that time, it is very likely they will never recover," says Kristy Murray of the University of Texas Health Science Center at Houston, the lead researcher on the study. Approximately $40 \%$ of patients monitored in the study continued to experience symptoms five years after infection.

Approximately 1 in 150 people infected by the West Nile virus develop a severe illness, such as West Nile encephalitis, according to the US Centers for Disease Control and Prevention. When the virus invades the nervous system during severe infections, the risk of long-term effects soars. "Those patients with [West Nile] encephalitis were less likely to recover than those who had meningitis or uncomplicated fever," says Murray.

There are currently no vaccines approved by the US Food and Drug Administration (FDA) against West Nile, but researchers and companies recognize the threat of the virus. "Protecting the elderly is one important goal of vaccine development," says Peter Mason, a professor of pathology at the University of Texas Medical Branch in Galveston and senior author on a rodent study published in May hinting that a DNA vaccine might help protect against the West Nile virus (Vaccine 26, 2762-2771; 2008).

The DNA vaccine developed by Mason uses a genetically modified virus that can only survive long enough to infect a single cell, and thus cannot cause disease. The engineered virus simply sticks around long enough to make the proteins needed to induce immune protection. Although promising, the vaccine is based upon an

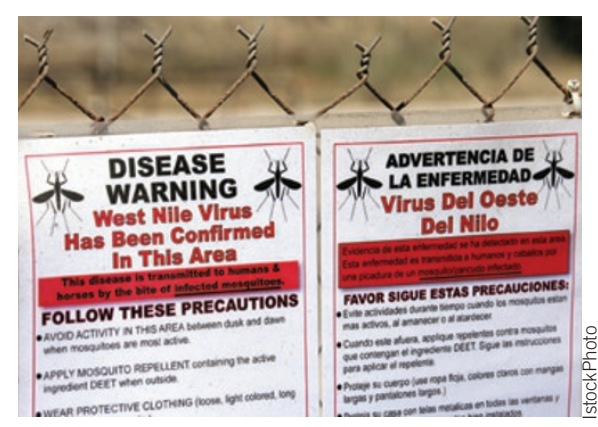

Warning signs: Symptoms can linger

approach that has not yet been approved by the FDA for any human vaccine.

Another vaccine, which uses a slightly more traditional approach, is also in the early stages of development. Researchers at the biotechnology company Acambis, headquartered in Cambridge, UK, have created a candidate vaccine by adding genes from the West Nile virus to a live attenuated virus derived from the yellow fever virus. In a small clinical study of human volunteers, $93 \%$ of participants given the vaccine candidate developed signs of an immune response (Proc. Natl. Acad. Sci. USA 103, 6694-6699; 2006).

Genevive Bjorn, Maui, Hawaii 